\title{
Interfacial Fermi level and surface band bending in Ni/semi-insulating GaAs contact
}

\author{
T. P. Chen, Y. C. Liu, S. Fung, and C. D. Beling \\ Department of Physics, University of Hong Kong, Hong Kong
}

(Received 22 March 1995; accepted for publication 19 June 1995)

\begin{abstract}
For nickel on the chemically clean surface of undoped semi-insulating $\mathrm{GaAs}$ at room temperature, an upward surface band bending of $0.062 \mathrm{eV}$ and a barrier height of $0.690 \mathrm{eV}$ have been observed by the photovoltage and the internal photoemission techniques, respectively. The observed surface band bending is in excellent agreement with its predicted value, and the observed barrier height also agrees very well with its value from the very careful analysis of reversed $I-V$ data. It has been determined that the interfacial Fermi level lies at $0.690 \mathrm{eV}$ below the GaAs conduction band minimum at the interface. The interfacial Fermi level is found to coincide with the energy level of the EL2 native defect, indicating the importance of the EL2 in the Fermi level pinning at the interface. (C) 1995 American Institute of Physics.
\end{abstract}

Semi-insulating (SI) GaAs is an important material which is currently used for fabricating high performance integrated circuits as well as for substrates for epitaxial layer growth. Although less attention has been paid to metal/SIsemiconductor contacts up to now, these contacts are nevertheless important in both technological and scientific aspects. For example, metal/SI-GaAs contact radiation detectors have recently received attention as charged particle and gamma ray spectrometers. ${ }^{1-3}$ The properties of these metal/ SI-GaAs contacts are obviously very important in determining the characteristics of the detectors. Although people at present know very little about Fermi level pinning at metal/ SI-semiconductor interfaces, studies on this issue will certainly be helpful for understanding Schottky barrier formation, which is still an unsolved problem in surface science. In contrast to the systems of metal on $n$ - and $p$-type semiconductors, which have been intensively investigated by conventional techniques including current-voltage $(I-V)$, capacitance-voltage $(C-V)$ and photoelectron spectroscopy, the systems of metal/Si-semiconductor are not easily accessible to these techniques due to the problems arising from the very low conductivity in SI semiconductors. In the present study, we show that the photovoltage and the internal photoemission techniques ${ }^{4,5}$ can be used for studying metal/SIsemiconductor contacts. The surface band bending and barrier height in the contact formed by $200 \AA$ Ni deposited on chemically clean surface of undoped SI-GaAs have been successfully measured by the two techniques.

The description of GaAs sample preparation can be found in Ref. 6, and the experimental setups for the photovoltage and internal photoemission measurements have been described in Refs. 4 and 5. In the photovoltage measurements, a monochromatic light beam with fixed energy of $1.70 \mathrm{eV}$ is focused onto the Ni/SI-GaAs contact so that the light is absorbed in the region close to the interface. It is observed that a photovoltage with the potential of the metal side higher than that of the semiconductor side is produced under the light illumination. From this polarity' of the photovoltage we conclude that there is a built-in electric field with the direction from the semiconductor side toward the metal side in the Ni/SI-GaAs contact. 'This means that there is an upward surface band bending in the contact. Therefore, the band diagram in the contact is similar to that in metal $/ n$ GaAs contacts, but the surface band bending in the contact is much smaller than that in a usual metal $n$-GaAs contact as shown below.

The internal photoemission technique is an accurate and direct method of determining the barrier height. As the surface band bending in the Ni/SI-GaAs is upward, the situation of pholoexcitation is similar to that in metal $/ n$-GaAs. When monochromatic light is incident upon the Ni film, photocurrent may be generated due to the photoexitation of electrons from the Fermi level in the Ni film to the GaAs conduction band. According to the Fowler theory, ${ }^{7}$ the square root of this photocurrent, $\sqrt{I_{p c}}$, is proportional to $\left(h \nu-q \Phi_{b}\right)$ for $\left(h \nu-q \Phi_{b}\right)>3 k T$, where $h \nu$ is the photon energy and $q \Phi_{b}$ is the barrier height measured from the Fermi level to the GaAs conduction band minimum (CBM) at the Ni/SIGaAs interface. When $\sqrt{I_{p c}}$ is plotted as a function of $h \nu, a$ straight line should be obtained, and the extrapolated value on the energy axis should directly give the barrier height. Figure 1 shows the square root $\sqrt{I_{\mathrm{pc}}}$ (in arbitrary units) of photocurrent normalized by unit incident photon flux in the $\mathrm{Ni} / \mathrm{SI}-\mathrm{GaAs}$ as a function of photon energy at room temperature. The intersection of the extrapolation with the energy axis yields a barrier height of $0.690 \mathrm{eV}$. Therefore, the position of the interfacial Fermi level $E_{f}^{i}$ (or the metal Fermi level) is determined to be $0.690 \mathrm{eV}$ below the $E_{c}^{i}$, i.e., $E_{f}^{i}=\left(E_{c}^{i}-0.690\right) \mathrm{eV}$, where $E_{c}^{i}$ is the CBM at the interface. The interfacial Fermi level coincides with the energy level of the native defect EL2 in GaAs which lies at $0.691 \mathrm{eV}$ below the CBM at the interface. ${ }^{8}$ This indicates the importance of the native defect EL2 in Fermi level pinning at the interface.

The above value of barricr height is in very good agreement with that determined from the reversed $I-V$ data. Figure 2 shows the reversed $I \sim V$ characteristics of the $\mathrm{Ni} / \mathrm{SI}-$ $\mathrm{GaAs}$ contact at room temperature. Note that in the following discussions the current and voltage are in absolute values. In a detailed study of the reversed $I-V$ characteristics of the contact, ${ }^{9}$ we found that, at small reversed bias $\left(0<V_{r} \leqslant 30 \mathrm{~V}\right)$, the reversed current is limited by the bulk 


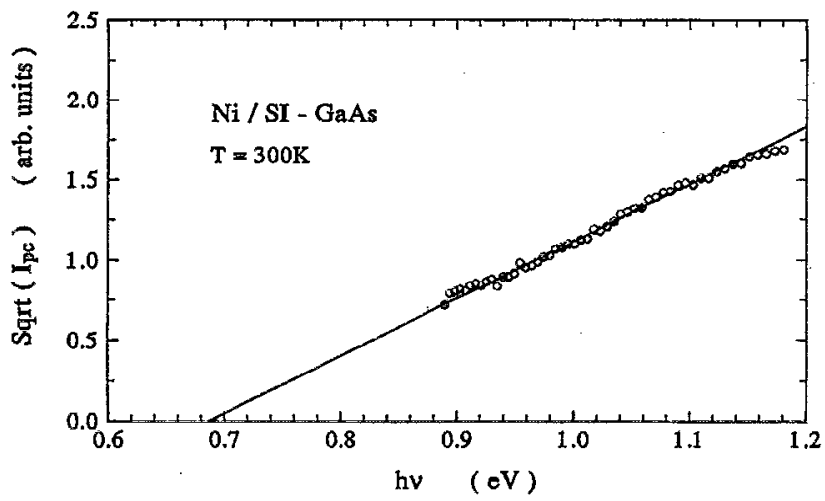

FIG. 1. Square root $\sqrt{I_{\mathrm{pc}}}$ (in arbitrary units) of photocurrent normalized by unit incident photon flux in Ni/SI-GaAs contact as a function of photon energy at room temperature.

resistance of the SI-GaAs and the $I-V$ relationship obeys the Ohmic rule,i .e., $V_{r}=I R$ with the bulk resistance $R=3.1 \times 10^{7}$ $\Omega$. However, at large reversed bias $\left(V_{r} \geqslant 50 \mathrm{~V}\right)$, the current is limited by thermionic emission at the Ni/SI-GaAs interface, and the reversed $I-V$ characteristics can be described by the thermionic emission theory ${ }^{10}$ as

$$
-I=I_{0}\left[\exp \left(-q V_{d} / k T\right)-1\right] \text {, }
$$

where the saturated current

$$
I_{0}=S A^{*} T^{2} \exp \left(-q \Phi_{b} / k T\right)
$$

and the voltage drop across the $\mathrm{Ni} / \mathrm{SI}-\mathrm{GaAs}$ contact is

$$
V_{d}=V_{r}-I R \text {. }
$$

In Eq. (2), $S$ is the area of the contact, and $A^{*}$ is the Richardson constant. For $q V_{d} \gg k T, I=I_{0}$. Therefore, as the reversed bias $V_{r}$ increases, the current $I$ saturates with the value of $I_{0}$. From Fig. 2, we obtain $I_{0}=1.1 \times 10^{-6} \mathrm{~A}$. The barrier height $q \Phi_{b}$ can thus be determined from Eq. (2). The value of the barrier height evaluated with $S=5.02 \times 10^{-5} \mathrm{~m}^{2}$ and $A^{*}=8.6 \times 10^{4} \mathrm{~A} \mathrm{~m}^{-2} \mathrm{~K}^{-2}$ is $0.691 \mathrm{eV}$. This value agrees very well with that obtained from the internal photoemission measurements.

Based on the above results, we are now able to estimate the surface band bending in the Ni/SI-GaAs contact. The compensation mechanism in the undoped SI-GaAs material

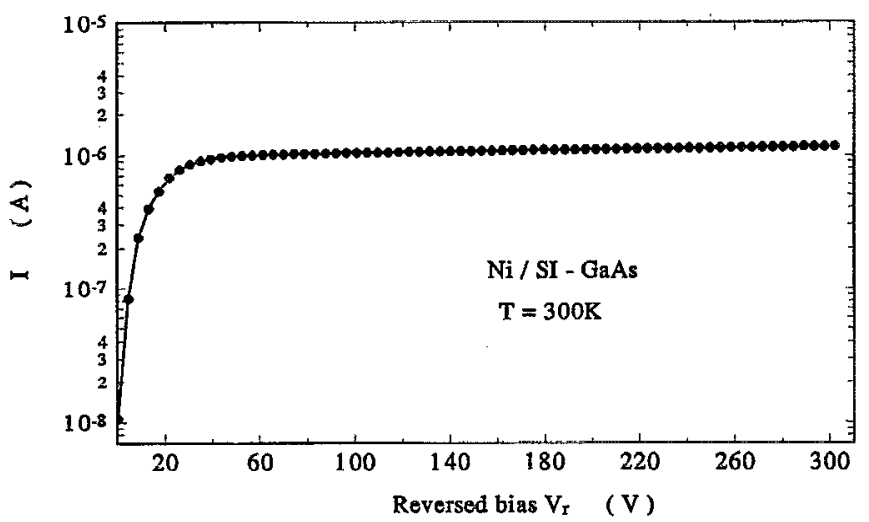

FIG. 2. Reversed $I-V$ characteristics of the Ni/SI-GaAs contact at room temperature.

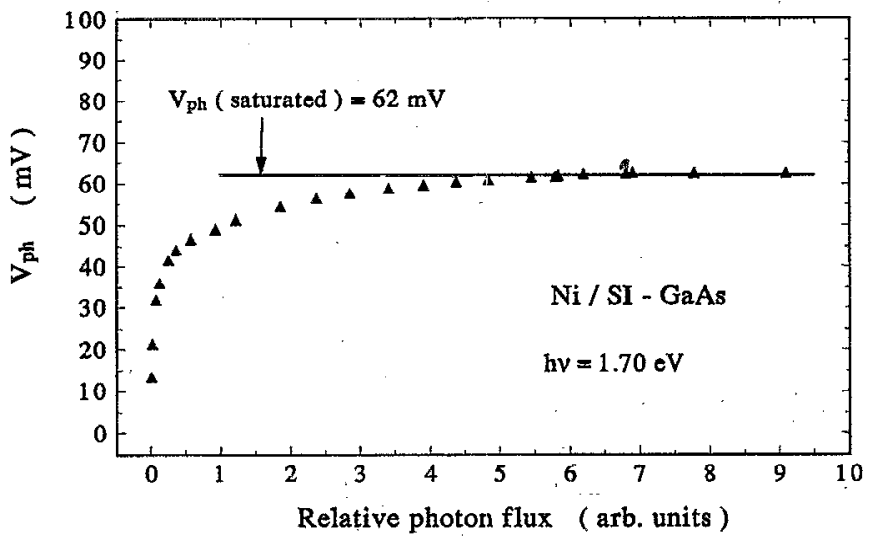

FIG. 3. Photovoltage generated in the Ni/SI-GaAs contact at room temperature under illumination with a fixed photon energy of $1.70 \mathrm{eV}$ vss relative incident photon flux.

used in the present study belongs to the case of deep donor/ shallow acceptor (DDSA). ${ }^{8}$ The deep donor is the native defect EL2 and the shallow acceptor is the residual carbon impurity. The Fermi level $E_{f}^{b}$, in the bulk of the SI-GaAs, can be determined as ${ }^{8}$

$$
E_{f}^{b}=E_{d d}-k T \ln g_{d d}+k T \ln \left(\frac{N_{d d}}{N_{s a}}-1\right)
$$

where $E_{d d}, g_{d d}$, and $N_{d d}$ are the energy level, the degeneracy factor, and the concentration for the deep donor (i.e., EL2), respectively, and $N_{s a}$ is the concentration for the shallow acceptor (i.e., the carbon impurity). For EL2, as given in Ref. $8, E_{d d}=E_{c}^{b}-0.691 \mathrm{eV}$ where $E_{c}^{b}$ is the CBM in the bulk SI-GaAs and $g_{d d}=0.845$. From the data sheet of the SI-GaAs material provided by the wafer supplier (CSI), the valucs of $N_{d d}$ and $N_{s a}$ are $1.5 \times 10^{16}$ and $1.5 \times 10^{15} \mathrm{~cm}^{-3}$, respectively. There may be some uncertainties in the two values, but they should not produce very significant influence on the result of the calculation of $E_{f}^{b}$ as the concentrations are involved in only the term $k T \ln \left(N_{d d} / N_{s a}-1\right)$ in Eq. (4). Using the above values of the relevant parameters in Eq. (4), we obtained at room temperature $E_{f}^{b}=E_{c}^{b}-0.629 \mathrm{eV}$. In thermal equilibrium, the whole system of Ni/SI-GaAs has a uniform Fermi level, and therefore

$$
E_{f}^{i}=E_{f}^{b} \text {. }
$$

From Eq. (5), it is found that $E_{c}^{i}-E_{c}^{b}=0.061 \mathrm{eV}$. This means that in thermal equilibrium an upward surface band bending of $0.061 \mathrm{eV}$ should exist in the Ni/SI-GaAs contact. This prediction has been confirmed quantitatively by the photovoltage measurements. As mentioned above, the surface band bending is upward. Figure 3 shows the photovoltage as a function of incident light intensity. At large light intensities the photovoltage saturates with a saturation value of $V_{\mathrm{ph}}$ (saturated) $=0.062 \mathrm{~V}$. The saturation of photovoltage indicates that the small surface band bending in the contact can easily be flattened by intensive light illumination at room temperature, and thus the value of the surface band bending is equal to the value of the saturated photovoltage in units of $\mathrm{eV}$, i.e., $E_{c}^{i}-E_{c}^{b}=q V_{\mathrm{ph}}$ (saturated) $=0.062 \mathrm{eV}$. This value is in excellent agreement with the above predicted value. 
In conclusion, the surface band bending and the barrier height in the Ni/SI-GaAs contact have been measured by the photovoltage and the internal photoemission techniques, respectively. A small upward surface band bending of $0.062 \mathrm{eV}$ and a barrier height of $0.690 \mathrm{eV}$ have been observed. The surface band bending obtained agrees very well with its predicted value and the observed barrier height is in very good agreement with the value determined from the careful analysis of reversed $I-V$ data, indicating that the two techniques can be reliably applied to metal/SI-semiconductor contacts. The Fermi level lies at $0.690 \mathrm{eV}$ below the conduction band minimum at the Ni/SI-GaAs interface, which coincides with the energy level of the native defect EL2 in GaAs, indicating the importance of EL2 in interfacial Fermi level pinning.
${ }^{1}$ D. S. McGregor, R. A. Rojeski, G. F. Knoll, F.L. Terry, Jr., J. Fast, and Y. Eisen, J. Appl. Phys. 75, 7910 (1994).

${ }^{2}$ T. J. Sumner, S. M. Grant, A. Bewick, J. P. Li, K. Smith, and S. P. Beaumont, Nucl. Instrum. Methods Scet. A 322, 514 (1992).

${ }^{3}$ K. W. Benz, R. Irsigler, J. Ludwig, J. Rosenzweig, K. Runge, F. Schäfer, J. Schneider, and M. Webel, Nucl. Instrum. Methods Sect. A 322, 493 (1992).

${ }^{4}$ T. P. Chen, T. C. Lee, S. Fung, and C. D. Beling, Semicond. Sci. Technol. 8, 2085 (1993).

${ }^{5}$ T. P. Chen, T. C. Lee, C. C. Ling, C. D. Beling, and S. Fung, Solid State Electron. 36, 949 (1993).

${ }^{6}$ T. C. Lee, Ph. D. thesis, The University of Hong Kong, 1993.

${ }^{7}$ R. H. Fowler, Phys. Rev. 38, 45 (1931).

${ }^{8}$ R. B. Darling, J. Appl. Phys. 74, 4571 (1993).

${ }^{9}$ T. P. Chen, Y. C. Liu, S. Fung, and C. D. Beling (unpublished).

${ }^{10}$ E. H. Rhoderick and R. H. Williams, Metal-Semiconductor Contacts, 2nd ed. (Clarendon, Oxford, 1988). 